\title{
Inhibition of IRF3-dependent antiviral responses by cellular and viral proteins
}

\author{
Tetsuo Tsuchida ${ }^{1}$, Taro Kawai ${ }^{1}$, Shizuo Akira ${ }^{1}$ \\ ${ }^{\prime}$ Laboratory of Host Defense, WPI Immunology Frontier Research Center, and Department of Host Defense, Research Institute for \\ Microbial Diseases, Osaka University, Japan \\ Cell Research (2009) 19:3-4. doi:10.1038/cr.2009.1; published online 5 January 2009
}

The host evokes innate immune responses to eliminate viruses by detecting the presence of infection. Host cells respond to nucleic acids derived from infected viruses to produce cytokines known as type I interferons (IFN $\beta$ and multiple IFN $\alpha$ ), which are the most important cytokines for host defense against viral infection. Type I interferons induce the synthesis of hundreds of IFN-inducible genes (ISG) that influence protein synthesis, growth arrest and cell death to induce the antiviral state as well as enhance dendritic cell maturation, natural killer cell activation, antibody production and differentiation of virus-specific cytotoxic $\mathrm{T}$ lymphocytes, which coordinately lead to the establishment of antiviral adaptive immune responses.

Innate immune detection of virus infection is mediated by host pattern recognition receptors (PRRs), including RNA helicases RIG-I and MDA5 and several members of Toll-like receptors (TLR) such as TLR3, TLR7 and TLR9 [1]. Of these, RIG-I, MDA5 and TLR3 function as sensors of double stranded RNA species that are produced by many viruses during replication, and trigger IFN $\beta$ induction. Transcription of the IFN $\beta$ gene requires the assembly of

Correspondence: Shizuo Akira

E-mail: sakira@biken.osaka-u.ac.jp three distinct families of transcription factors, including NF- $\kappa \mathrm{B}$, ATF2/c-Jun and IRF3, to the positive regulatory domains (PRD) in the promoter region; and signals by RIG-I/MDA5 and TLR3 activate these three transcription factors [2]. Of these, IRF3 plays a critical role in the initial induction of the IFN $\beta$ gene. IRF3 is a constitutively expressed protein that resides in the cytoplasm in unstimulated cells, but virus infection triggers IRF3 phosphorylation at serine/ threoine clusters in the C-terminal regulatory regions, which allows IRF3 to form a homodimer, translocate into the nucleus and bind to DNA. RIG-I/MDA5 and TLR3 signaling pathways converge on a common TRAF3 adapter complex, which then activates two protein kinases TBK1 and IKKi that function as IRF3 kinases.

Conjugation of proteins by ubiquitin is fundamentally important in various cellular processes, including innate immune signaling [3]. Ubiquitin contains seven lysine residues that could mediate the formation of polyubiquitin chains. Lysine 48 (K48)-linked polyubiquitination typically targets proteins for degradation by the ubiquitin-proteasome pathway while K63-linked polyubiquitination modulates protein-protein interactions and protein cellular localization to influence the activation of signaling pathways. Moreover, cellular deubiquitinating (DUB) enzymes catalyze the removal of polyubiquitin chains to counteract the effects of ubiquitination. Two studies by Zheng et al. and Zhang et al. in a recent issue of Cell Research identified new cellular and viral proteins, both of which negatively regulate type I IFN induction by modulating IRF3 ubiquitination but with different mechanisms $[4,5]$.

The study of Zhang et al. demonstrated that the E3 ubiquitin ligase $\mathrm{RBCC}$ protein interacting with $\mathrm{PKC} 1$ (RBCK1) binds to IRF3 and targets it for ubiquitination and subsequent degradation through a proteasome-dependent pathway. Consistently, overexpression of RBCK1 suppressed Sendai virusinduced type I IFN induction, while knockdown of RBCK1 resulted in enhanced type I IFN induction. Notably, they found that Sendai virus infection increased the level of RBCK1 expression, which was accompanied by a decrease in IRF3 levels. They therefore concluded that RBCK1-mediated IRF3 degradation is important for termination of IRF3-dependent antiviral responses. Although type I IFN is required for elimination of viruses, aberrant type I IFN production contributes to the development of autoimmune disorders, such as systemic lupus erythematosus [6]. Thus, mechanisms that suppress the production of type I IFN are critical to maintain homeostasis.

Several cellular factors have been 
suggested to participate in negative regulation of IRF3. These include cytoplasmic peptidyl-prolyl-isomerase Pin1 and Ro52/TRIM21 [7, 8]. Pin1 binds phosphorylated IRF3 to trigger its ubiquitination and subsequent degradation. However, unlike RBCK1, PIN1 expression is constitutive and it interacts with IRF3 after stimulation. Whereas PIN1 does not affect NF$\kappa \mathrm{B}$ activation, RBCK1 has a role in suppressing NF- $\kappa B$ activation. These findings suggest that RBCK1 plays a broader role in the suppression of host antiviral responses than PIN1. Ro52/ TRIM21, which is an inducible protein and an autoantigen present in patients with systemic lupus erythematosus and Sjögren's syndrome, also targets IRF3 for ubiquitination and degradation. However, it also interacts with other IRFs such as IRF7 and IRF8, in contrast to RBCK1 that specifically binds IRF3 but not IRF7. Moreover, Ro52/TRIM21 enhances IRF8-dependent inflammatory cytokine expression via K63-linked ubiquitination rather than degradation [9]. Thus, Ro52/TRIM21 positively and negatively regulates various IRFs.

To escape from host innate immune responses, viruses have acquired mechanisms that antagonize or inhibit innate immune receptor-triggered type I IFN induction, as a result of co-evolution between host and viruses. Zheng et al. sought to analyze evasion mechanisms of the mouse hepatitis virus A59 (MHVA59), which belongs to coronaviruses, a family that also includes members such as severe acute respiratory syndrome (SARS) coronavirus. They found that IRF3 is ubiquitinated, and this ubiquitination is required for its nuclear translocation rather than degradation. They demonstrated that Papain-like protease domain 2 (PLP2) of nonstructural protein 3 (nsp3), which contains conserved deubiquitinase (DUB) motif, binds to IRF3 and causes its de-ubiquitination to prevent its nuclear localization, thereby suppressing IFN $\beta$ production. Moreover, they provided evidence that IRF3 is modified by K48- and K63linked polyubiquitin chains, and PLP2 de-ubiqutinates both types via its DUB activity. Thus, the DUB activity of PLP2 is responsible for coronavirus inhibition of host type I IFN induction. A recently identified cellular DUB, DUBA, is capable of inhibiting TRAF3 activation, thus suppressing IRF3 activation [10]. PLP2 may have a similar role to DUBA, although future study will be required to investigate whether PLP2 suppresses TRAF3 activation.

Taken together, these two studies suggest that IRF3 activation is positively and negatively regulated by ubuquitination. Virus infection may trigger K48- and K63-linked ubiquitination of IRF3 in the cytoplasm, which is prerequisite for promoting its nuclear translocation. In the nucleus, IRF3 ubiquitination (perhaps K48-linked) may be linked to degradation that is required for termination of type I IFN responses. IRF3 plays a key role in virus infection-induced type I IFN production. Thus, inhibition of IRF3 activation is an efficient way to terminate type I IFN responses. In this respect, many viruses have acquired mechanisms that suppress IRF3 activation to escape from host type I IFN effects. Thus, control of IRF3 activation via ubiquitination will be a target for designing therapeutic strategies against viral infection and autoimmune disorders.

\section{References}

1 Kawai T, Akira S. Innate immune recognition of viral infection. Nat Immunol 2006; 7:131-137.

2 Honda K, Takaoka A, Taniguchi T. Type I interferon [corrected] gene induction by the interferon regulatory factor family of transcription factors. Immunity 2006; 25:349-360.

3 Liu YC, Penninger J, Karin M. Immunity by ubiquitylation: a reversible process of modification. Nat Rev Immunol 2005; 5:941-952.

4 Zhang M, Tian Y, Wang RP, et al. Negative feedback regulation of cellular antiviral signaling by RBCK1-mediated degradation of IRF3. Cell Res 2008; 18:1096-1104.

5 Zheng D, Chen G, Guo B, Cheng G, Tang H. PLP2, a potent deubiquitinase from murine hepatitis virus, strongly inhibits cellular type I interferon production. Cell Res 2008; 18:1105-1113.

6 Baccala R, Hoebe K, Kono DH, Beutler B, Theofilopoulos AN. TLR-dependent and TLR-independent pathways of type I interferon induction in systemic autoimmunity. Nat Med 2007; 13:543-51.

7 Saitoh T, Tun-Kyi A, Ryo A, et al. Negative regulation of interferon-regulatory factor 3-dependent innate antiviral response by the prolyl isomerase Pin1. Nat Immunol 2006; 7:598-605.

8 Higgs R, Ní Gabhann J, Ben Larbi N, et al. The E3 ubiquitin ligase Ro52 negatively regulates IFN-beta production post-pathogen recognition by polyubiquitin-mediated degradation of IRF3. J Immunol 2008; 181:1780-1786.

9 Kong HJ, Anderson DE, Lee CH, et al. Cutting edge: autoantigen Ro52 is an interferon inducible E3 ligase that ubiquitinates IRF-8 and enhances cytokine expression in macrophages. $J$ Immunol 2007; 79:26-30.

10 Kayagaki N, Phung Q, Chan S, et al. DUBA: a deubiquitinase that regulates type I interferon production. Science 2007; 318:1628-1632. 